\title{
Are Brain Organoids Equivalent to Philosophical Zombies?
}

\author{
Mainá Bitar \\ QIMR Berghofer Institute, Australia \\ Maina.Bitar@qimrberghofer.edu.au
}

\begin{abstract}
Along just over a century of research we moved from learning how to cultivate tissues in a dish to grasping the concepts for creating an entire brain in a vat. As we approach the divisive moment in which we can first detect signs of awareness in such artificially developed organoids, we need to lay foundation for what lays ahead. It is crucial that ethical, legal and moral implications of organoid research are clear and that boundaries are set to separate scientific progress from human life preservation. The largest obstacle may be the definition of consciousness itself, which has arguably been historically neglected by philosophy, psychology and neurosciences at large. One reason may be the difficulties posed by the underlying qualities of awareness, such as its subjective and heterogeneous nature. Another reason may lie on the possibly that consciousness is an overarching emergent property of our brain. For the time being, one can see brain organoids as philosophical zombies, physical analogues of the human brain which mimic sentient human reactions but lack experiential properties of sensation (a.k.a. qualia).
\end{abstract}

Keywords: brain organoids; stem cells; brain in a vat 


\section{Historical Background}

Progress in the field of organoids is not linear, as is the norm in science, and recent years have witnessed an extraordinary increase in the complexity and number of studies (Figure 1). Nevertheless, the first attempts of explanting tissue from animals and growing it in culture for further observations were made over a century ago. Johns Hopkins University had a pivotal role, incidentally bringing together Ross Granville Harrison and Thomas Montrose Burrows. Harrison was not the first to venture on tissue grafting, but was arguably the first to succeed. In his experiment, Harrison isolated the medullary plate of frogs and used rudimentary tissue culture techniques to show the growth of neurites without many additional factors [1]. Burrows was a medical student at the time of these iconic experiments and, after both of them left Johns Hopkins, Burrows went to visit Harrison in his Yale laboratory. At Yale, Burrows learned the technique he then (allegedly) termed 'tissue culture', the principles of which are still a main part of laboratory life throughout the world today. On his return, Burrows carried on tissue culture experiments during his fellowship at Rockefeller University, supervised by Alexis Carrel. Together, Burrows and Carrel made significant improvements to the technique (e.g. [2,3]) and managed to maintain their cultures for over three decades, therefore claiming to have derived immortal lines (although others have further revisited this claim, e.g. [4]).

Even after the extraordinary progress that followed these first steps we summarized, until the beginning of the present century, the possibility of isolating human skin cells and using these as substrate to create a heterogeneous, three-dimensional, functional, cortical structure was difficult to contemplate and seemed purely speculative. Although, in many ways, tissue culture had become more sophisticated than when first developed, there was one main feature which remained mostly unchanged: cell cultures were essentially two-dimensional and homogeneous. In just a few years this has changed significantly and in many laboratories today adult skin fibroblasts are routinely reprogrammed into pluripotent stem cells, which then give rise to a myriad of cell types. One of the most ingenious developments of this basic principle is the adaptation of commercial ink jet printers 
to produce growth factor arrays (by substituting the ink cartridges with a solution containing specific growth factors) in which cells are grown and induced to various levels of differentiation [5] Applying similar methods, still in the two-dimensional space, researchers were able to recapitulate physiological and morphological aspects of the neocortex, using signaling molecules to lead stem cells into becoming cortical precursors (reviewed in [6]).

The first steps towards the three-dimensional space were establishing new culture techniques and further making these serum-free [7-10]. Forthcoming studies (e.g. [11]) then combined these three-dimensional cell aggregates with elements of the extracellular matrix, frequently in the form of a scaffolding substance (such as Matrigel or hydrogel). Intriguingly, these studies revealed that, in the appropriate environment, the same pluripotent cells derived from human skin are capable of self-organizing and originating miniaturized organ-like structures.

\section{The First of Brain Organoids}

Incremental progress has culminated in the landmark publication by Lancaster and her collaborators describing the emergence of three-dimensional cerebral organoids from human pluripotent cells [12]. Growing up to a few millimeters, the structured neural tissue showed evidence of compartmentalized elements with specialized regions which seemed to recapitulate elements of the complex human brain closer than ever before. Clusters reminiscent of specialized brain regions were observed and, although these were discrete, they were not completely isolated, showing evidence of communication. In additional to an accurate cellular composition of its different regions, organoids can also develop individualized functional synapses, although these do not seem to form a proper circuitry and do not exhibit myelination (further reviewed in [6]). Impressively, these systems needed just over a week (8-10 days) to develop their neural identity and an extra two to three weeks (20-30 days in total) for defined brain regions to emerge. Nevertheless, what invariably happened afterwards was that these organoids would reach a size limit overtime, which was likely defined by the absence of blood vessels, impairing oxygen and nutrient exchange 
(advances are expected in the near future that will allow proper oxygen and nutrient diffusion in brain organoids, mimicking vascularization; [13]).

The use of human brains or even tissues derived from adult or embryonic human brains is subject to obvious ethical and social restrictions (for comprehensive reviews of the topic, readers are referred to [14] and specially the comment article [15], although many others have tackled the subject). On the other hand, as so many characteristics are inherent of the Homo sapiens species and not mirrored in other mammals, it is important to appreciate the unique advantages of being able to investigate biological phenomena in the context of organoids derived from human cells in replacement of e.g. murine models. For example, the outer region of the subventricular zone (oSVZ), a structure drastically expanded in our species, significantly contributes to the speciesspecific increased size and complexity of the human cortex [16]. The oSVZ is populated by intermediate progenitors and unique stem cells named outer radial glia (oRGs), which previous research showed can be produced in vitro from human progenitor stem cells (e.g. [7]). One of the findings of Lancaster and her collaborators [12] is that, in their cerebral organoid models, oRGs reside in oSVZ-like progenitor regions, mimicking the in vivo localization. The oRGs are present in rodents to a minimum and completely absent in mice, which may help explain the failure of murine models to recapitulate the markedly reduced brain of humans with microcephaly. In contrast, the cerebral organoid model stablished by Lancaster et al. is capable of following human brain development, in particular cortex development. Although this is a well known example in which authors assessed underlying factors of microcephaly, many other diseases are exclusive of human beings (or at least have exclusive features which only emerge in humans). Organoid studies are a valid, and now broadly considered, alternative for exploring human disease in an in vivo-like context.

\section{Brain in a Vat and Philosophical Zombie Hypotheses}

A philosophical zombie [18] is a conceptual subject which is physically undistinguishable 
from human being but lacks consciousness and thus is not sentient (i.e. does not possess qualia). When a philosophical zombie is, for instance, cut by a knife, it will not experience pain but it will react and exactly as if it had. This behavior will therefore be merely physical and no awareness is related to it. There is no feeling of internal suffering because there is no mind but there is a body, which reacts mimicking the way a human would. In this case we know, by definition, there is no consciousness.

Philosophers such as Hilary Putnam [19] have addressed conceptual experiments derived from the 'brain in a vat' context, in which a brain is removed from the body to which it was once attached, kept alive by artificial means and connected to a supercomputer. The machine would then provide the brain with electrical impulses that would mimic the usual stimuli the brain is physiologically subjected to. Although there is an entire field of philosophy dedicated to what unfolds in this hypothetical scenario, dating back to Descartes and the Cartesian skepticism, here we will limit discussion to one simple question: would a brain in a vat constitute a conscious entity or not?

Until recently, a debate of this nature would be merely theoretical and the context was only illustrated in so-called thought experiments. In a very recent publication, a team of scientists led by Nenad Sestan [20] successfully restored molecular and cellular functionality of a porcine brain hours after death. With their elegant and complex system, carefully designed to minimize the damaging effects of death upon the brain, researchers managed to preserve cytoarchitecture, prevent cell death and restore synaptic activity, among other hallmarks of a living brain. The study, however, has raised multiple questions, both ethical and philosophical, as well as a debate upon the legal and medical definitions of death. A lengthy feature article in The New York Times Magazine [21] is an example of the broad reach of the study, in which Sestan speculated on future impacts and raised relevant concerns about the lack of guidelines and restrictions for research to follow (specially regarding possible replicates of the experiment in human brains). Although results were remarkable to say the least, there was no restoration of consciousness and we are likely far from witnessing that 
milestone, if we ever do. Nonetheless there are many aspects one can appreciate when reflecting on the implications of this proof of principle experiment.

\section{Organoids as Philosophical Zombies}

In parallel, as research on brain organoids advances, it will arise similar questions to Sestan's work and it is crucial that, as governments, scientists and human beings, we prepare for what is likely to come as we explore these new avenues. As previously discussed here (and as others reviewed elsewhere), organoids are robust and complex systems which successfully recapitulate some of the fundamental microscopic and macroscopic structures of different human organs. Initially, as mentioned, the development of fully functional independent organoids seemed doomed to be restricted by the lack of an efficient nutrient and oxygen delivery system. However, recent advances in the field aim to couple different systems into multidimensional structures that better represent the interconnected human body. One can foresee the development of tubular networks through which nutrients can be infused to the system. Others will attempt to integrate organoids of different organs together, thus laying foundation to the emergence of entire systems which will eventually be able to communicate [22]. In this scenario, one can connect brain organoids with cardiac organoids, intestinal organoids and etc. In this sense we are moving towards the emergence of mini-brains (which would represent a step further from brain organoids).

The stimulation of cerebral organoids has been shown to replicate simple sensations such as patterned synaptic connections (e.g. [10]), response to light exposure [23] and orchestrated network activity [24]. On the other hand, most of the similarities between cerebral organoids and the developing brain have only been reported at the molecular and cellular level and higher-order patterns of a conscious brain were not yet observed [25]. As discussed by Lavazza and Massimini [26], one of the most important tasks ahead of us is to clearly define the boundaries between biological materials and conscious systems.

As research in cerebral organoids gradually moves forward, these currently peppercorn- 
sized structures $(\sim 5 \mathrm{~mm})$ more closely resemble miniature brains in vats, we revisit the question we posed before: would a brain organoid constitute a conscious entity or not?

\section{Defining Consciousness}

To even begin to tackle this question, one must first and foremost be able to define consciousness, in a precise and measurable way. A conundrum that certainly arises is that brain organoids are themselves a mean to study consciousness. Therefore, limiting research on brain organoids based solely on supposed evidence of a primordial consciousness may hinder the very subject of defining consciousness. We can lay this paradoxical thought aside, at least for the sake of argument, if we believe scientist are able to define consciousness based on data from non-invasive (or minimally invasive) procedures, such as electroencephalography, functional magnetic resonance imaging, behavioral tests, etc. In this line, even though we can argue to have made significant advance, we are not less mesmerized and overwhelmed by the subject than William James was in the nineteenth century. James was a philosopher and psychologist, dubbed by many to be the father of American psychology. Upon addressing the understanding of the correspondence between 'brain states' and 'mind states' (i.e. the connection between physiological states of the brain and conscious manifestations of the individual, also known as identity theory), he affirmed this would be "the scientific achievement, before which all past achievements would pale" [27]. Advancing nearly one hundred years, it has been a few decades since Francis Crick ventured into neuroscience (perhaps a comparatively little known fact, Crick spent his last 25 years studying the brain), and he concluded that "it is remarkable that most of the work in both cognitive science and the neurosciences makes no reference to consciousness (or 'awareness')" [28]. Some researchers today use this essay from Crick and Christof Koch to mark the origin of a second wave in the study of consciousness.

Intriguingly, there is a line of modern philosophy which either denies the existence of such connection between the abstract concept of consciousness and the physical world or denies that this can ever be accurately assessed. Philosophers who support this view reject the mind-brain identity 
theory, specially in its generalized form (that all mental states - qualia - are identical with physical states). Such debate is not the scope of this work, although we appreciate the arguments to sustain this rejection of the identity theory. However, several observations argue against this view and maybe the most compelling evidence is the fact that consciousness can be localized within the brain (to the posterior cortex). Patients who suffer trauma resulting in the spinal cord being severed become paralyzed but given no substantial damage occurred to their nervous system elsewhere, they still experience most senses and are able to feel, think, remember events, express their personality and report their sense of self. By all means these are all examples of conscious individuals. The same is true for patients that had their cerebellum removed or lost or were even born without it. More extraordinary perhaps is the fact that the removal of even large portions of the frontal cortex also does not seem to have significant impact in consciousness. Taken all of this together and adding to the opposite observation that removing even small portions of the posterior cortex leads to impairments in consciousness, scientists have at times delimited this area as the place of consciousness, as Melanie Boly and her collaborators have reviewed [29].

This view, in which consciousness can consequently be defined as the set of properties regulated by the posterior cortex can arguably be deemed reductionist. One important notion that particularly undermines this disseminated theory of a 'localized consciousness' is emergence. Emergent properties arise in a system but cannot be detected in any of its individual elements. It is the very confluence of all parts that allow for these properties to become present, which is not observed in segregated parts. We may argue that consciousness is an emergent property of the brain and indeed this seems to explain many aspects of it. This view was likely introduced by the work of Roger Sperry [30] in which he defines consciousness not as one functional outcome of the brain, but as a "holistic form of control over the flow pattern of cerebral excitation" (i.e. if we imagine the brain as an orchestra, consciousness would be the maestro rather than the string section). One important piece of evidence supporting this theory comes from recent findings which characterize whole-brain awareness [31]. In the study, while subjects viewed an image briefly flash on a screen, 
scientists observed an increase in the intermodular functional connectivity of the brain network in detriment of a modular response. Their findings point to global alterations on the cerebral functional connectivity underlying awareness.

\section{Measuring Consciousness}

From the attempts made to design reliable tools to measure consciousness levels in individuals (or even brain organoids in the future), the most comprehensive and applicable is perhaps the one developed by a team led by Marcello Massimini and Giulio Tononi [32]. Using intense magnetic stimulation to incite electrical signals through the cortex, scientists recorded the patterns of brain activity using electroencephalogram sensors. Observations showed an inverse relationship between the complexity of such patterns and the level of consciousness (i.e. the more predictable the patterns, the closer the subject was to being unconscious). A data-compression algorithm was then cleverly applied to estimate the complexity of each set of records. These estimates were used to defined a perturbation complexity index (PCI). After applying the measurements to 150 individuals ranging from fully awake and conscious to completely unconscious, the team derived a reliable scale in which a person can be deemed conscious if their PCI is over 0.31. The method was successful in further assessing consciousness in a set of noncommunicative patients who were either minimally conscious or in vegetative state, surprisingly identifying 9 of the 43 patients in vegetative state as conscious to some extent.

A general theoretical definition is that the conscious experience is both informative and integrative. In this sense the PCI would represent an objective quantitative measure, only reaching higher levels when the response is widespread (which indicates it is integrative) and diversified (which indicates it is informative). As variations of such measurement strategies adapted to in vitro systems are already being tested, these could be implemented in future versions of brain organoids.

\section{Ethical, Philosophical and Moral Implications}


The ability to experience pain is also generally seen as a hallmark of consciousness. In this sense, there is an inherent need to develop tests for identifying sentient states in brain organoids. Although a major and obvious advantage of developing organoids (for any organ for that matter) is to circumvent the use of mammalian animal models, which we know can experience pain, being able to detect (even a rudimentary) response to pain in mini-brains would theoretically promote debate on the subject. Suffering in animal models is often aggravated by confinement and general deprivation of freedom (suppression of hunting and mating instincts, limitation of movement, restricted diet, etc.), while this may not be an immediate concern for research performed in organoids, if some level of consciousness is detected in these systems, then suffering may be an underlying experience. In this sense, some of the guidelines for the use of animal models in scientific studies can also be applied for research on brain organoids (e.g. the principles of the 3Rs, replacement, reduction and refinement [33]).

Another question posed by the event of finding evidence of higher levels of functionality or even consciousness in brain organoids is how should we then dispose of this biological material (this may not even be an acceptable term in that context). In many countries, when miscarriage happens prior to 5-6 months of pregnancy, there is no specific law or regulation in place for how to properly handle the fetuses. This may raise concern about the proper protocol for discarding brain organoids when these get to the same level as brains of preterm babies (at $~ 25-37$ weeks of gestation). On the other hand, if stillbirth (i.e. when the fetus dies after at least 5 months of pregnancy) occurs, the general rule (frequently enforced by law) is that the infant should either be buried or cremated and parents may be entitled to a specific form of death certificate. As we anticipate brain organoids to soon be able to mimic at least some features of fetal brains, regulation should be in place to specifically define how research material should be disposed, preserving the legal rights of parents who experience miscarriage or stillbirth.

In parallel, if cerebral organoids, or mini-brains, ever reach a point in which we are able to detect levels of awareness, consciousness and/or manifestations of pain and suffering regulations 
must be in place to enable research without violation of human rights. Before such organoids can mimic the brain activity of not only fetuses, but also comatose patients, or patients with locked-in syndrome [34], we will need to carefully design criteria to assess all aspects of life which may overlap with organoid research in order to fully preserve human rights.

Although organoid research is arguably still far from reaching this point in time, other aspects can already be subject of concern. Recently, different groups of researchers have successfully transplanted cerebral organoids built from human neural stem cells into mice (e.g. $[35,36])$ resulting in enhanced integration to the surrounding tissue (e.g. vast vascularization) and promoting maturation and differentiation. An unexpected development, however, was the observation of graft-to-host synaptic connectivity, with the growth of human axonal projections extending into the mice brain. Previous researchers led by Steven Goldman have introduced human glial cells in mice brains and observed that the transplanted cells have actively replaced the host murine cells overtime, resulting in a mostly humanized glial population. Moreover, the chimeric mice showed enhanced long-term potentiation and increased synaptic plasticity, culminating in superior cognitive performance measured by a variety of learning tasks [37]. The study was laying ground for further research on human disorders such as schizophrenia [38], but raised much concern from the public as to whether these chimeric mice could be intellectually compared to humans to some extent.

Given the possible future implications of brain organoids, it has been suggested that research on them should be restricted or prohibited, due to an inherent moral status [39]. There is a subjective moral value attributed to organized three-dimensional structures composed of human cells (e.g. brain organoids), which surpasses the level of the biological material (e.g. [40]). A general rule has been proposed based on the assumption that cerebral organoids are comparable to early developing human embryos in some aspects and could therefore be considered under the same ethical agreements. This could lead to limiting organoid complexity to the stage of embryos and halt their further progress into structures resembling brains in fetal stage. 
Objectively, brain organoids may be subject to limitations somewhat similar to the '14-day rule', a restriction based on a point that defines the time after which embryos develop a neural system and individual identity (since it cannot lead to twins thereafter). Others have carried similar discussions on the moral status of brain organoids, proposing to transfer definitions and regulations already in place for other contexts in which the levels of consciousness are very near the threshold of non-existence, as in patients in (pseudo)comatose states [26,41]. This would not come without a compromise of course and one could argue such decision may not be in the best interest of science.

In summary, this is a controversial matter, inevitably associated with the development of human brain organoids and many aspects are yet to be debated before any conclusion can ever be reached. At the present moment, I believe it is as important to lay ground for future regulations as it is to carry on technical improvements to the available organoid systems (at least while these are still somewhat rudimental images of actual brains). As much as the beneficial impacts of this developing field for human health (and the understanding of human development and evolution) are tremendous, its legal, moral and ethical implications are still largely unpredictable. Although no degree of conscious-like activity can be accurately detected in brain organoids yet, it is necessary that we are able to precisely measure levels of awareness and pain, in order to define the limits within which we would like to keep any applications of research on these mini-brains. For now, we may be only dealing with philosophical zombies, but further advances may change this, specially if we witness the emergence of artificially created awareness. Finally, I would like to echo the words of Sestan when commenting about his 2019 groundbreaking experiment: "Everyone of these decisions should not be up to us [scientists] alone. It is up to all of us, we make these decisions together."

\section{Acknowledgements}

I thank Dr. Guy Barry for his valuable insight in to this manuscript. 


\section{Conflicts of Interest}

Authors declare no conflicts of interest.

\section{References}

1. Harison, R.G. Observations on the living developing nerve fibe. Proc Soc Exp Biol Med 1906, 4(1), 140-143

2. Burrows, M.T.; Carrel A. Cultivation of tissues in vitro and its technique. J Exp Med 1911, 13(3), 387-96

3. Burrows, M.T.; Carrel A. An addition to the technique of the cultivation of tissues in vitro. J Exp Med 1911, 14(3), 244-247

4. Witkowski, J. Dr. Carrel's immortal cells. Medical history 1980, 24(2), 129-42

5. Watanabe, K.; Fujiyama, T.; Mitsutake, R.; Watanabe, M.; Tazaki, Y.; Miyazaki, T.; Matsuda, R. Fabrication of growth factor array using an inkjet printer. Cell and Organ Printing 2010, 203-222

6. Kelava, I.; Lancaster, M.A. Dishing out mini-brains: Current progress and future prospects in brain organoid research. Developmental Biology 2016, 420(2), 199-209

7. Watanabe, K.; Kamiya, D.; Nishiyama, A.; Katayama, T.; Nozaki, S.; Kawasaki, H.; et al. Directed differentiation of telencephalic precursors from embryonic stem cells. Nat Neurosci 2005, 8, 288-296

8. Eiraku, M.; Watanabe, K.; Matsuo-Takasaki, M.; Kawada, M.; Yonemura, S.; Matsumura, M.; et al. Self-organized formation of polarized cortical tissues from ESCs and its active manipulation by extrinsic signals. Cell Stem Cell 2008 , $3,519-532$

9. Mariani, J.; Simonini, M.V.; Palejev, D.; Tomasini, L.; Coppola, G.; Szekely, A.M.; et al. Modeling human cortical development in vitro using induced pluripotent stem cells. Proc Natl Acad Sci 2012, 109, 12770-12775

10. Paşca, A.M.; Sloan, S.A.; Clarke, L.E.; Tian, Y.; Makinson, C.D.; Huber, N.; et al. Functional cortical neurons and astrocytes from human pluripotent stem cells in 3D culture. Nat Methods 2015, 12, 671-678

11. Lindborg, B.A.; Brekke, J.H.; Vegoe, A.L.; Ulrich, C.B.; Haider, K.T.; Subramaniam, S.; Venhuizen, S.L.; Eide, C.R.; Orchard, P.J.; Chen, W.; Wang, Q.; Pelaez, F.; Scott, C.M.; Kokkoli, E.; Keirstead, S.A.; Dutton, J.R.; Tolar, J.; O'Brien, T.D. Rapid induction of cerebral organoids from human induced pluripotent stem cells using a chemically defined hydrogel and defined cell culture medium. Stem Cells Translational Medicine 2016, 5(7), 970-979

12. Lancaster, M.; Renner, M.; Martin, C.; Wenzel, D.; Bicknell, L.S.; Hurles, M.E.; Homfray, T.; Penninger, J.M.; Jackson, A.P.; Knoblich, J.A. Cerebral organoids model human brain development and microcephaly. Nature 2013, 501, 373-379

13. McMurtrey R.J. Analytic Models of oxygen and nutrient diffusion, metabolism dynamics, and architecture optimization in three-dimensional tissue constructs with applications and insights in cerebral organoids. Tissue Eng Part C Methods 2016, 22(3):221-49

14. Ramos-Zúñiga R.; González-Pérez O.; Macías-Ornelas A.; Capilla-González V.; Quiñones-Hinojosa A. Ethical implications in the use of embryonic and adult neural stem cells. Stem Cells Int. 2012, 470949.

15. Farahany, N.; Greely, H.; Hyman, S.; Koch, C.; Grady, C.; Paşca, S.; Sestan, N.; Arlotta, P.; Bernat, J.; Ting, J.; Lunshof, J.; Iyer, E.; Hyun, I.; Capestany, B.; Church, G.; Huang, H.; Song, H. The ethics of experimenting with human brain tissue. Nature 2018, 556, 429-432

16. Hansen, D.; Lui, J.; Parker, P.; Kriegstein, A. Neurogenic radial Glia in the outer subventricular zone of human neocortex. Nature 2010, 464, 554-561

17. Shi, Y.; Kirwan, P.; Livesey, F. Directed differentiation of human pluripotent stem cells to cerebral cortex neurons and neural networks. Nature protocols 2012, 7, 1836-46 
18. Kirk, R. Sentience and Behaviour. Mind 1974, 83, 43-60

19. Putnam, H. Brains in a vat. In Reason, Truth and History. Cambridge University Press 1981, 1-21

20. Vrselja, Z.; Daniele, S.G.; Silbereis, J.; Talpo F.; Morozov, Y.M.; Sousa, A.M.M.; Tanaka, B.S.; Skarica, M.; Pletikos, M.; Kaur, N; Zhuang, Z.W.; Liu, Z.; Alkawadri, R'; Sinusas, A.J.; Latham, S. R.; Waxman, S.G.; Sestan, N. Restoration of brain circulation and cellular functions hours post-mortem. Nature 2019, 568, 336-343

21. Shaer, M. Scientists are giving dead brains new life. What could go wrong? The New York Times Magazine 2019

22. Chukwurah E.; Osmundsen A.; Davis S.W.; Lizarraga S.B. All together now: Modeling the interaction of neural with non-neural systems using organoid models. Front Neurosci, 2019, 13, 582

23. Quadrato, G.; Nguyen, T.; Macosko, E.Z.; Sherwood, J.L.; Yang, S.M.; Berger, D.R.; Maria, N.; Scholvin, J.; Goldman, M.; Kinney, J.P.; Boyden, E.S.; Lichtman, J.W.; Williams, Z.M.; McCarroll, S.A.; Arlotta, P. Cell diversity and network dynamics in photosensitive human brain organoids. Nature 2017, 545, 48-53

24. Trujillo, C.A.; Gao, R.; Negraes, P.D.; Gu, J.; Buchanan, J.; Preissl, S.; Wang, A.; Wu, W.; Haddad, G.G.; Chaim, I.A.; Domissy, A.; Vandenberghe, M.; Devor, A.; Yeo, G.W.; Voytek, B.; Muotri, A.R. Complex oscillatory waves emerging from cortical organoids model early human brain network development. Cell Stem Cell 2019, 25(4), 558-569

25. Qian X.; Song, H.; Ming, G. Brain organoids: advances, applications and challenges. Development 2019, 146

26. Lavazza, A.; Massimini, M. Cerebral organoids and consciousness: How far are we willing to go? Journal of Medical Ethics 2018, 44(9), 613-614

27. William, J. The Principles of Psychology. Published by Henry Holt and Company 1918

28. Crick, F.; Koch, C. Towards a neurobiological theory of consciousness. Seminars in the neurosciences 1990, 2, 263275.

29. Boly, M.; Massimini, M.; Tsuchiya, N.; Postle, B.R.; Koch, C.; Tononi, G. Are the neural correlates of consciousness in the front or in the back of the cerebral cortex? Clinical and neuroimaging evidence. Journal of Neuroscience 2017, 37(40), 9603-9613

30. Sperry, R.W. A modified concept of consciousness. Psychological Review 1969, 76(6), 532-536

31. Godwin, D.; Barry, R.L.; Marois, R. Breakdown of the brain's functional network modularity with awareness. Proc Natl Acad Sci 2015, 112(12) 3799-3804

32. Casarotto, S.; Comanducci, A.; Rosanova, M.; Sarasso, S.; Fecchio, M.; Napolitani, M.; Pigorini, A.G.; Casali, A.; Trimarchi, P.D.; Boly, M.; Gosseries, O.; Bodart, O.; Curto, F.; Landi, C.; Mariotti, M.; Devalle, G.; Laureys, S.; Tononi, G.; Massimini, M. Stratification of unresponsive patients by an independently validated index of brain complexity. Ann Neurol 2016, 80(5), 718-729

33. Wolfensohn, S.; Hawkins, P.; Lilley, E.; Anthony, D.; Chambers, C.; Lane, S.; Lawton, M.; Robinson, S.; Voipio, H.; Woodhall, G. Reducing suffering in animal models and procedures involving seizures, convulsions and epilepsy. $\mathbf{J}$ Pharmacol Toxicol Methods 2012, 67(1), 9-15

34. Laureys, S.; Pellas, F.; Eeckhout, P.; Ghorbel, S.; Schnakers, C.; Perrin, F.; Berre, J.; Faymonville, M.; Pantke, K.; Damas, F.; Lamy, M.; Moonen, G.; Goldman, S. The locked-in syndrome: What is it like to be conscious but paralyzed and voiceless?. Progress in brain research 2005, 150, 495-511

35. Daviaud N.; Friedel R.H.; Zou H. Vascularization and Engraftment of Transplanted Human Cerebral Organoids in Mouse Cortex. eNeuro 2018 5(6)

36. Mansour, A.; Gonçalves, J.; Bloyd, C.W.; Li, H.; Fernandes, S.; Quang, D.; Johnston, S.; Parylak, S.L.; Jin, X.; Gage, F.H. An in vivo model of functional and vascularized human brain organoids. Nat Biotechnol 2018, 36, 432441

37. Windrem, M.S.; Schanz, S.J.; Morrow, C.; Munir, J.; Chandler-Militello, D.; Wang, S.; Goldman, S.A. A competitive advantage by neonatally engrafted human glial progenitors yields mice whose brains are chimeric for 
human glia. Journal of Neuroscience 2014, 34(48), 16153-16161

38. Windrem, M.S.; Osipovitch, M.; Liu, Z.; Findling, R.L.; Tesar, P.J.; Goldman S.A. Human iPSC glial mouse chimeras reveal glial contributions to schizophrenia. Cell Stem Cell 2017, 21, 195-208

39. Hostiuc, S.; Rusu, M.C.; Negoi, I.; Perlea, P.; Dorobanţu, B.; Drima, E. The moral status of cerebral organoids. Regenerative Therapy 2019, 10, 118-122

40. Boers, S.N.; de Winter-de Groot, K.M.; Noordhoek, J.; Gulmans, V.; van der Ent, C.K.; van Delden, J.J.M.; Bredenoord, A.L. Mini-guts in a dish: Perspectives of adult Cystic Fibrosis (CF) patients and parents of young CF patients on organoid technology. Journal of Cystic Fibrosis 2018, 17(3), 407-415

41. Shepherd, J. Ethical (and epistemological) issues regarding consciousness in cerebral organoids. Journal of Medical Ethics 2018, 44(9)

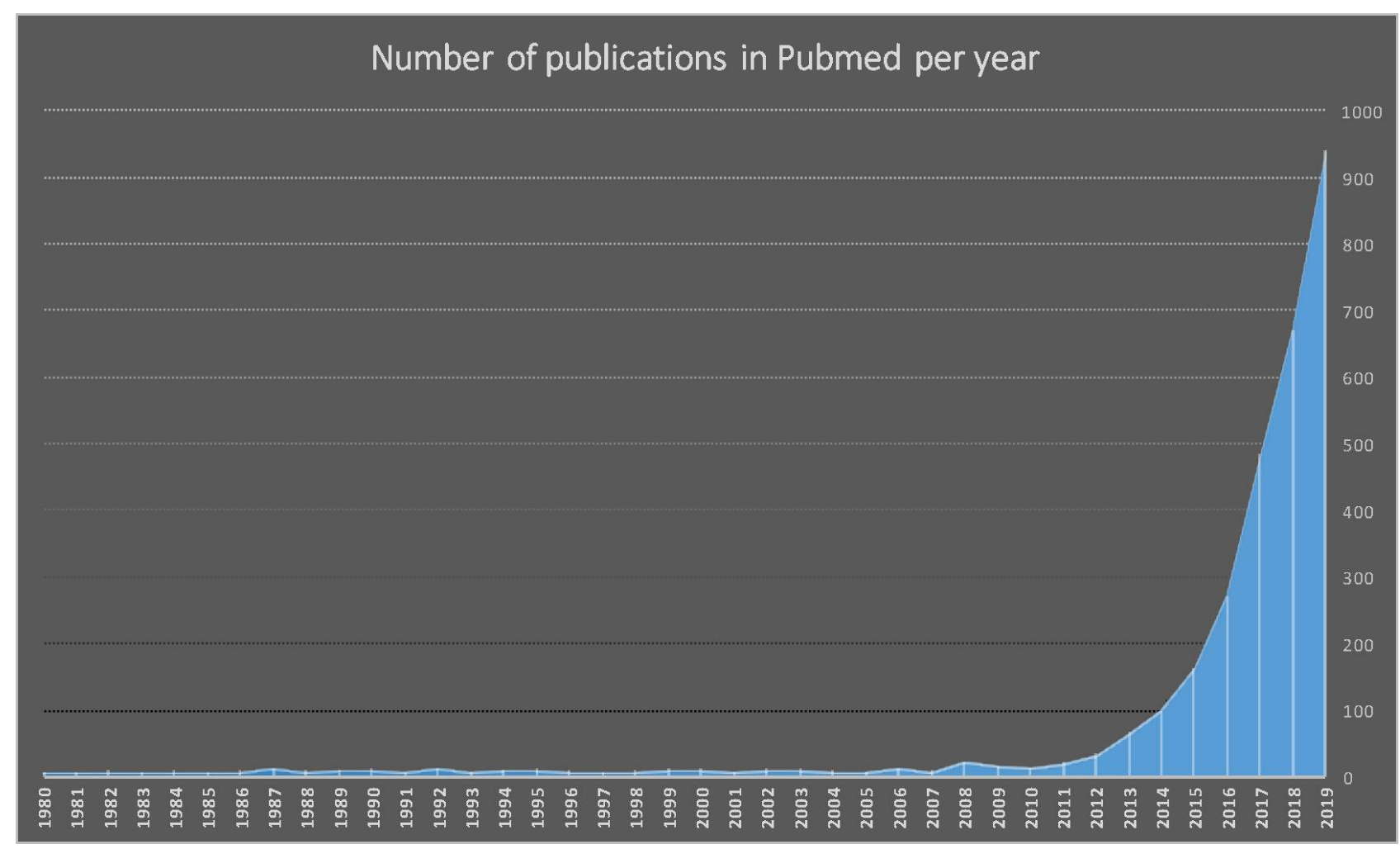

Figure 1: Publications containing the term 'organoids' in either their title or abstract from 1980 to 2019. Results were retrieved from the public literature using search engines for scientific articles. 